and nintedanib are licensed treatments but are only available at specialised centres. We have previously shown that patients whose local hospital was a prescribing centre (PC) were more likely to be receiving AFDs than those whose local hospital was not a prescribing centre (NPC). ${ }^{1}$

Objectives We set out to test the hypothesis that this indicates differences in ability to travel or to seek specialist care which might be reflected in differences in indices of multiple deprivation (IMD).

Methods We obtained a full list of patients who received AFDs since 2013 and obtained their postcodes from hospital databases. We additionally obtained markers of socio-economic status based on the IMD score obtained from government websites. Data were recorded in January 2016 and July 2017 and compared with non-parametric statistics.

Results The number of patients per 100000 population in each postcode area started on AFDs increased from a median (range) of $3.04(0-15.86)$ in 2016 to $8.81(1.16-33.87)$ in $2017\left(\mathrm{p}=4 \times 10^{-6}\right)$. In both $2016(\mathrm{p}=0.0119)$ and 2017 $(p=0.0089)$, there were more patients on AFDs per postcode area where the local hospital was a PC compared to a NPC. Looking at the distribution of IMD in 2017, there was a small difference $(p=0.057)$ that did not appear fully to explain the difference in AFD prescriptions between PC and NPC.

Conclusions The NHS constitution requires equality in access to therapy regardless of where the patient lives. Although AFD prescriptions have increased significantly between 2016 and 2017, we have again demonstrated inequality of access to AFDs depending on patient location. These differences do not appear fully explained by differences in indices of multiple deprivation.

Refernce 1. Woodhead FA, Townsend S, Desai D. P171 Health inequality exists in pirfenidone prescription for idiopathic pulmonary fibrosis in the English Midlands according to patient location. Thorax 2016, Dec 1;71(3):A176-7.

\section{M27 EFFECT OF PIRFENIDONE ON BREATHLESSNESS AS MEASURED BY THE UCSD-SOBQ SCORE IN PATIENTS WITH IDIOPATHIC PULMONARY FIBROSIS (IPF) WITH MODERATE LUNG FUNCTION IMPAIRMENT}

${ }^{1} \mathrm{MK}$ Glassberg, ${ }^{2} \mathrm{M}$ Wijsenbeek, ${ }^{3} \mathrm{~F}$ Gilberg, ${ }^{4} \mathrm{U}$ Petzinger, ${ }^{3} \mathrm{KU}$ Kirchgaessler, ${ }^{5} \mathrm{C}$ Albera. ${ }^{1}$ University of Miami Health System, Florida, US; ${ }^{2}$ Erasmus University Medical Centre, Rotterdam, Netherlands; ${ }^{3}$ F. Hoffman-La Roche Ltd, Basel, Switzerland; ${ }^{4}$ Accovion GmbH, Eschborn, Germany; ${ }^{5}$ University of Turin, Turin, Italy

\subsection{6/thoraxjnl-2017-210983.449}

Introduction Treatment of IPF with pirfenidone slows disease progression as measured by changes in forced vital capacity (FVC), independent of baseline FVC values. In a previous analysis of patients with limited vs more advanced lung function impairment, increases in University of California, San Diego Shortness of Breath Questionnaire (UCSD-SOBQ) scores were more pronounced in patients with Gender Age Physiology index (GAP) stage II/III vs GAP stage I and in patients with baseline FVC $<80 \%$ vs FVC $\geq 80 \% 0^{-1}$ We examined the effect of pirfenidone on UCSD-SOBQ in these subpopulations. Methods 1247 patients in ASCEND (NCT01366209) and CAPACITY (NCT00287716; NCT00287729) were randomised to pirfenidone $2403 \mathrm{mg} / \mathrm{d}$ or placebo. Patients were stratified by GAP stage I vs stage II/III and by baseline\%-predicted FVC. The effect of pirfenidone on UCSD-SOBQ score was

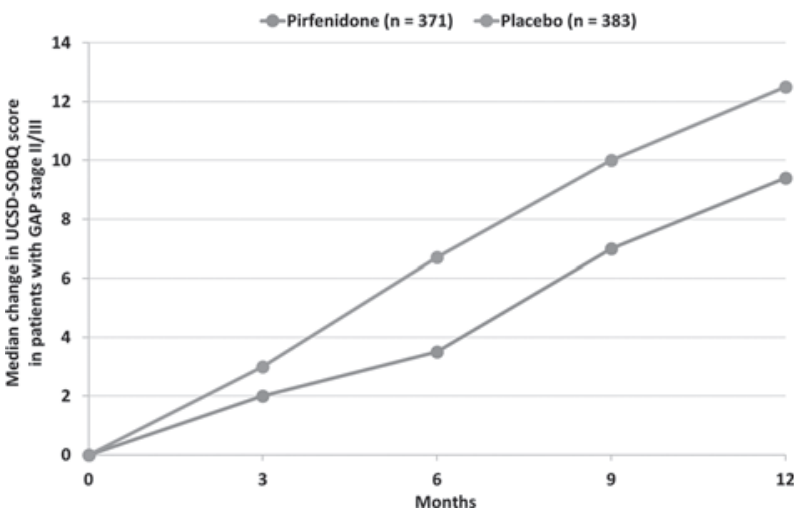

Abstract M27 Figure 1 Median increase in UCSD-SOBQ scores from baseline in patients with GAP stage II/III.

assessed by continuous and categorical changes from baseline over 12 months, and by multiples of the minimal clinically important difference of 5 points for UCSD-SOBQ.

Results Pirfenidone-treated patients with GAP stage II/III had higher UCSD-SOBQ scores after 12 months than those with GAP stage I (median increase from baseline: 9.4 vs 5.0); similar Results occurred with placebo (12.5 vs 4.3). GAP stage II/ III patients treated with pirfenidone had less increase in median UCSD-SOBQ score at 12 months compared with those receiving placebo (9.4 vs 12.5 ; median difference -3.5 , 95\% CI $-6.2,-0.5 ; \mathrm{p}=0.0161)$ with the curves diverging after 3 months (figure 1). Evaluation of categorical change for patients with GAP stage II/III demonstrated that pirfenidone reduced the proportion of patients with UCSD-SOBQ score increases of $\geq 15$ points $(45.6 \%$ vs $38.4 \%$; $p=0.0449)$ and $\geq 20$ points $(37.7 \%$ vs $28.6 \%$; $=0.0089)$ at 12 months compared with placebo; increases of $\geq 5$ or $\geq 10$ points were similar between treatment groups. Results in patients with $\%$ FVC $\leq 80 \%$ were comparable to GAP stage II/III.

Conclusions In patients with IPF with moderate lung function impairment, pirfenidone reduced the progression of breathlessness compared with placebo. Patients receiving pirfenidone showed less change from baseline in UCSD-SOBQ score and a lower proportion of patients had more pronounced increases in UCSD-SOBQ scores at 12 months.

\section{REFERENCE}

1. Albera C et al. Eur Respir J 2016;48:843-851.

\section{M28 DEFERRING TREATMENT WITH PIRFENIDONE RESULTS IN LOSS OF LUNG FUNCTION THAT IS NOT RECOVERED BY LATER TREATMENT INITIATION}

${ }^{1} \mathrm{~T}$ Maher, ${ }^{2} \mathrm{~S}$ Jouneau, ${ }^{3} \mathrm{~L}$ Morrison, ${ }^{4} \mathrm{D}$ Lederer, ${ }^{5} \mathrm{M}$ Molina-Molina, ${ }^{6} \mathrm{~K}-\mathrm{U}$ Kirchgaessler, ${ }^{6} \mathrm{~F}$ Gilberg, ${ }^{6} \mathrm{~J}$ Axmann, ${ }^{7} \mathrm{U}$ Petzinger, ${ }^{8} \mathrm{E}$ Bendstrup. ${ }^{1}$ Royal Brompton Hospital and Imperial College London, London, UK; ${ }^{2}$ Hôpital Pontchaillou, IRSET UMR 1085, Université de Rennes 1, Rennes, France; ${ }^{3}$ Department of Medicine, Duke University, Durham, NC, US; ${ }^{4}$ Columbia University Medical Centre, New York, NY, US; ${ }^{5}$ University Hospital of Bellvitge, Institut d'Investigacions Biomèdiques de Bellvitge, Barcelona, and Centro de Investigación Biomédica en Red Enfermedades Respiratorias (CIBERES), Madrid, Spain; ${ }^{6}$ F. Hoffmann-La Roche Ltd., Basel, Switzerland; ${ }^{7}$ Accovion GmbH, Eschborn, Germany; ${ }^{8}$ Department of Respiratory Diseases and Allergy, Aarhus University Hospital, Nørrebrogade 44, 8000 Aarhus, Denmark

10.1136/thoraxjnl-2017-210983.450 